\title{
Population Pharmacokinetics of Magnesium Sulfate in Preeclampsia and Associated Factors
}

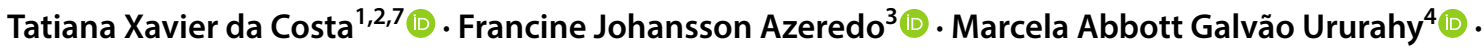 \\ Miguel Adelino da Silva Filho ${ }^{5} \cdot$ Rand Randall Martins $^{6} \cdot$ Antonio Gouveia Oliveira $^{6}$ (B)
}

Published online: 8 July 2020

(c) The Author(s) 2020

\begin{abstract}
Background and Objective The pharmacokinetic basis of magnesium sulphate $\left(\mathrm{MgSO}_{4}\right)$ dosing regimens for preeclampsia (PE) prophylaxis and treatment is not clearly established. The aim of study is to develop a population pharmacokinetic (PK) model of $\mathrm{MgSO}_{4}$ in $\mathrm{PE}$, and to determine key covariates having an effect in $\mathrm{MgSO}_{4}$ pharmacokinetics in preeclampsia (PE) and to determine key covariates having an effect in $\mathrm{MgSO}_{4} \mathrm{PK}$.

Methods A prospective cohort study was conducted from June 2016 to February 2018 in patients with PE administered $\mathrm{MgSO}_{4}$ as a 4-g bolus followed by continuous infusion at a rate of $1 \mathrm{~g} / \mathrm{h}$. Serum magnesium concentrations were obtained before treatment administration and 2,6,12, and $18 \mathrm{~h}$ after the initial dose. The software Monolix was used to estimate population PK parameters of $\mathrm{MgSO}_{4}$ [clearance $(\mathrm{CL})$, volume of distribution $(V)$, half-life] and to develop a PK model with baseline patient demographic, clinical, and laboratory covariates.

Results The study population consisted of 109 patients. The PK profile of $\mathrm{MgSO}_{4}$ was adequately described by a one-compartment PK model. The model estimate of the population CL was $1.38 \mathrm{~L} / \mathrm{h}$; for $V$, it was $13.3 \mathrm{~L}$; and the baseline magnesium concentration was $0.77 \mathrm{mmol} / \mathrm{L}(1.87 \mathrm{mg} / \mathrm{dL})$. The baseline body weight and serum creatinine statistically influenced $\mathrm{MgSO}_{4}$ $\mathrm{CL}$ and $V$, respectively. The model was parameterized as CL and $V$.

Conclusion The $\mathrm{PK}$ of $\mathrm{MgSO}_{4}$ in pregnant women with PE is significantly affected by creatinine and body weight. Pregnant women with $\mathrm{PE}$ and higher body weight have a higher $V$ and, consequently, a lower elimination rate of $\mathrm{MgSO}_{4}$. Pregnant women with $\mathrm{PE}$ and a higher serum creatinine value show lower $\mathrm{CL}$ and, therefore, lower $\mathrm{MgSO}_{4}$ elimination rate.
\end{abstract}

\section{Introduction}

Preeclampsia (PE) is a multifactorial disease belonging to the spectrum of hypertensive syndromes of pregnancy and

Tatiana Xavier da Costa and Antonio Gouveia Oliveira contributed equally to this work.

Francine Johansson Azeredo, Marcela Abbott Galvão Ururahy, Miguel Adelino da Silva Filho, Rand Randall Martins also contributed equally to this work.

Tatiana Xavier da Costa

tatycx1000@gmail.com

1 Postgraduate Program in Pharmaceutical Sciences, Health Science Center, Federal University of Rio Grande do Norte, Natal, Brazil

2 Maternity School Januário Cicco, Health Science Center, Federal University of Rio Grande do Norte, Natal, Brazil

3 Post Graduate Program in Pharmacy, Faculty of Pharmacy, Federal University of Bahia, Salvador, Brazil is characterized by elevated blood pressure and proteinuria after 20 weeks of gestation [1,2]. Recent definitions also include organ dysfunction, such as kidney failure, hepatic failure, hematologic complications, uteroplacental dysfunction, and fetal neurological growth restriction [1]. Eclampsia is the convulsive form of PE and affects $0.1 \%$ of all pregnant women [3]. In The United States, PE and eclampsia are among the four leading causes of maternal mortality, with a maternal death rate of 1:100,000 live births [4]. However, in Africa and Asia, PE is responsible for up to $9 \%$ of maternal

4 Department of Clinical Analysis, Health Science Center, Federal University of Rio Grande do Norte, Natal, Brazil

5 Health Department, Uninassau University, Parnamirim, Brazil

6 Department of Pharmacy, Health Science Center, Federal University of Rio Grande do Norte, Natal, Brazil

7 Present Address: Faculty of Pharmacy, Health Science Center, Federal University of Rio Grande do Norte, Natal, Brazil 


\section{Key points}

Magnesium sulfate is an effective and low-cost medication used to treat seizures in pre-eclampsia.

Despite the widespread use of magnesium sulfate, there are no individualized administration protocols for pregnant women with pre-eclampsia.

Through population pharmacokinetics, clinical characteristics with potential for dose individualization were identified.

deaths, and in Latin America and the Caribbean, it is responsible for nearly $25 \%$ of maternal deaths [5].

Magnesium sulfate $\left(\mathrm{MgSO}_{4}\right)$ is the drug of election to prevent seizures in PE. The World Health Organization considers that $\mathrm{MgSO}_{4}$ is safe and cost-effective in the prophylaxis of seizures and in the treatment of severe PE and eclampsia $[5,6]$. Since the therapeutic serum magnesium concentration level is not yet well established, the administration rate of $\mathrm{MgSO}_{4}$ is monitored by the regular surveillance of signs and symptoms of magnesium toxicity, which include loss of the patellar reflex, blurred vision, drowsiness, slurred speech, muscle paralysis, and respiratory depression [7]. This is a rather crude method of controlling the dosage of $\mathrm{MgSO}_{4}$, a drug with a narrow therapeutic margin, with doses too low increasing the risk of convulsions, while doses too high can result in maternal/fetal toxicity. Therefore, pharmacokinetic (PK) and pharmacodynamic (PD) data are key to establishing optimized dosage regimes for this drug [8].

Population PK studies aim to develop a model that can identify sources of the variability in drug concentration in the patient population. Population PK seeks to identify the measurable pathophysiologic factors that cause changes in the dose-concentration relationship and the extent of these changes so that if such changes are associated with clinically significant shifts in the therapeutic index, dosage can be appropriately modified $[9,10]$. The effect estimates of patient characteristics on drug PK obtained with the model may be used in clinical practice to optimize pharmacotherapy [9]; however, only a very small number of published reports have studied the population $\mathrm{PK}$ of $\mathrm{MgSO}_{4}$ in $\mathrm{PE}$ $[8,11,12]$. Therefore, the aim of this study was to develop a population $\mathrm{PK}$ model of $\mathrm{MgSO}_{4}$ in $\mathrm{PE}$ and to identify patient factors influencing the $\mathrm{PK}$ of this drug.

\section{Patients and Methods}

\subsection{Study Design and Population}

This study was approved by the ethics committee of the Onofre Lopes University Hospital (number 49959215.8.0000.5292). All procedures performed in this study involving human participants were in accordance with the ethical standards of the institutional research committee and with the 1964 Declaration of Helsinki. Informed consent was obtained from all the individual participants included in the study.

This was an observational, longitudinal, prospective study to evaluate the population $\mathrm{PK}$ of $\mathrm{MgSO}_{4}$ in $\mathrm{PE}$ and associated factors, conducted between June 2016 and February 2018 at the maternal intensive care unit (ICU) of the Januário Cicco Maternity School, a six-bed ICU with an average of 70 admissions per month. All pregnant women admitted to the ICU with PE confirmed by clinical and laboratory criteria, with an indication for therapy with $\mathrm{MgSO}_{4}$, without any exclusion criteria, and who gave written informed consent were included in the study. Exclusion criteria were extreme and morbid obesity (body mass index $>40 \mathrm{~kg} / \mathrm{m}^{2}$ ), decompensated diabetes mellitus with a serum glucose $>300 \mathrm{mg} / \mathrm{dL}$ ), gemellar pregnancy, delivery scheduled for less than $18 \mathrm{~h}$ after the end of $\mathrm{MgSO}_{4}$ infusion, and contraindication to the use of $\mathrm{MgSO}_{4}$ (myasthenia gravis or other neuromuscular disorder, renal insufficiency, hypermagnesemia, hypocalcemia, hypokalemia, recent myocardial infarction, and atrioventricular conduction defect).

\subsection{Administration of Magnesium Sulfate}

$\mathrm{MgSO}_{4}$ was administered according to the Zuspan protocol (a loading dose of $4 \mathrm{~g}$, equivalent to $16 \mathrm{mmol}$ of $\mathrm{MgSO}_{4}$, given intravenously in $30 \mathrm{~min}$, followed by a maintenance dose of $\mathrm{MgSO}_{4}$ at an infusion rate of $1 \mathrm{~g} / \mathrm{h}$ ) [13]. Serum magnesium concentrations were obtained immediately before the loading dose and $2 \mathrm{~h}, 6 \mathrm{~h}, 12 \mathrm{~h}$, and $18 \mathrm{~h}$ thereafter. Demographic data (sex, age, weight, height) and clinical (serum creatinine, total protein and albumin concentration, comorbidities, and concomitant medications) were obtained at baseline.

\subsection{Bioanalytical Analysis}

The method used for the dosage of $\mathrm{MgSO}_{4}$ was colorimetric and based on the Mann and Yoe principle. Mann and Yoe dye in alkaline $\mathrm{pH}$ and in the presence of magnesium develops a red coloration. The red color intensity of the complex 
is proportional to the concentration of magnesium. Magnesium is measured at an absorbance of $500 \mathrm{~nm}$.

\subsection{Development of the Population PK Model (POPPK Analysis)}

The PK parameters of $\mathrm{MgSO}_{4}$ were estimated using a population approach using Monolix Software ${ }^{\circledR}$ Suite 2018R1 (Lixoft $^{\circledR}$, Antony, France). Population PK analysis was based on the principles outlined by the Food and Drug Administration (FDA) and the European Medicines Agency (EMA) $[10,14]$.

Based on studies available in the literature [15], the different models, such as one- and two-compartment with first order elimination rate, were evaluated. Residual errors were also tested in order to choose the best model to fit the data. No endogenous magnesium baseline adjustment was made to the model.

The model used to determine the plasma concentration versus time after testing various possibilities was the following:

$C \mathrm{p}=\frac{k_{0}}{\mathrm{CL}} \cdot\left(e^{k_{\mathrm{e}} \cdot T}-1\right) \cdot e^{-k_{\mathrm{e}} \cdot t}$,

where $C \mathrm{p}$ is the plasma concentration, $k_{0}$ is the infusion rate, $\mathrm{CL}$ is the clearance, $k_{\mathrm{e}}$ is the elimination constant, $t$ is the time in which $C$ p is estimated, and $T$ is the infusion time. $k_{\mathrm{e}}$ can also be determined as:

$k_{\mathrm{e}}=\frac{\mathrm{CL}}{V}$,

where $k_{\mathrm{e}}$ is the elimination constant, $\mathrm{CL}$ is the clearance, and the $V$ is the volume of distribution.

The observed plasma concentration data were used to predict population parameters, and individual and interindividual variability (IIV). For IIV, the parameters of the $i$ subjects $\left(\theta_{i}\right)$ were described using a log-normal distribution as follows:

$\theta_{i}=\theta \exp \left(\eta_{i}\right)$

where $\theta$ is the typical population parameter and $\eta_{i}$ is the individual random effect of an approximately normal distribution with mean zero and variance $\varpi^{2}$ to describe the parameter IIV. As a covariate can explain some of the IV in one parameter, the set of individual parameters $\left(\Theta_{i}\right)$ is given according to the typical parameters of the population $(\Theta)$, individual values of covariates $\left(c_{i}\right)$, and random effects $\left(\eta_{i}\right)$ :

$\Theta_{i}=g\left(\Theta, c_{i}, \eta_{i}\right)$.

The final model was parameterized as $\mathrm{CL}$ and $V$. The parameters were estimated with a stochastic approach.
Different error models were analyzed to explain the variability of the residual error, including a constant, proportional, and combined error model. The models were compared considering the likelihood values, represented by $-2 \times \log$-likelihood (-2LL) and the Akaike information criteria (AIC), the selected model being the one presenting lower values of those statistics as well as graphic criteria for better model fit.

\subsection{Analysis of Covariates}

The covariates were tested after the base model was established. First, an analysis of the data was performed to identify the covariates associated with magnesium concentration over time and the correlation between both continuous and categorical covariates, and the PK parameters $\mathrm{CL}$ and $V$ were computed. These generated plots of the random effect residues $(\eta)$ versus the covariates. The covariates investigated in the selected base model were serum levels of albumin, total protein and creatinine, age, gestational age, weight, body mass index, presence of comorbidities (previous hypertension and infectious disease), and concomitant use of other drugs (Dipirona, hydralazine, methyldopa, nifedipine). Total proteins, albumin, and creatinine were chosen as covariates, as they are parameters that may be related to PK, representing the transport and elimination of medications. Age and weight are factors related to the patient that can influence PK. The presence of previous hypertension and medications most used in hospitalization were chosen as covariates, as they are factors that could interfere with the $\mathrm{PK}$ of $\mathrm{MgSO}_{4}$. The ones that showed statistically significant association $(p<0.05)$ with CL and/or $V$ were added to the final model.

\subsection{Model Performance and Validation}

The internal validity of the model was assessed with a bootstrap approach using the package Rsmlx for RStudio software (version 1.1.442, Free Software Foundation, Boston, USA). The analysis data were sampled with replacement to generate 1000 replicates and estimates of the median parameter, and 95\% confidence intervals were obtained.

To evaluate model adjustment, the predictions using the developed model were compared to the observed data, and model adjustment was evaluated by visual predictive check (VPC). This is a graph that displays the 5th, 50th, and 95th percentiles of the observed data and the data predicted by the final model. The VPC (500 repetitions) was performed based on the parameters of the final model POPPK, comparing the distribution of simulated POPPK data (median, 95\% confidence interval) against the observed data. The VPC of the final model was performed with the initialization parameters estimated. The VPCs were also stratified by subject, infusion duration, and other relevant key covariates (e.g., 
$V, \mathrm{CL})$ to evaluate the predictive properties of the observed data model.

\section{Results}

\subsection{Study Population}

The population PK model was obtained from 109 participants, in whom three to four determinations of plasma concentration were obtained, resulting in a total of 347 data points. The patients had a mean age of $25.8 \pm 7.4$ years, and all were in the third trimester of pregnancy. Baseline magnesium values were $1.9 \pm 0.6 \mathrm{mg} / \mathrm{dL}$ before the beginning of the administration. Most patients had previous pregnancies (66\%) (Table 1).

\subsection{Development and Evaluation of the Population PK Model (POPPK)}

The one-compartment structural model was chosen based on the analysis of the tested models (Table 2), and it is in agreement with some studies available in the literature [15]. One can verify that the developed model has the ability to predict the individual and population plasma concentrations for most patients evaluated. From the visual analysis, a reduced plasma concentration decay after the end of

Table 1 Characteristics of the study population $(n=109)$

\begin{tabular}{ll}
\hline Parameters & Values \\
\hline Age in years (mean $\pm \mathrm{SD})$ & $25.8 \pm 7.4$ \\
Body weight in kg (mean $\pm \mathrm{SD})$ & $79.2 \pm 14.7$ \\
Baseline magnesium in mg/dL (mean $\pm \mathrm{SD})$ & $1.9 \pm 0.6$ \\
Gestational age in weeks $($ mean $\pm \mathrm{SD})$ & $35.2 \pm 4.2$ \\
Baseline creatinine in mg/dL $($ mean $\pm \mathrm{SD})$ & $0.7 \pm 0.3$ \\
Number of pregnancies, $n(\%)$ & \\
One & $48(44.4)$ \\
Two & $24(22.2)$ \\
Three or more & $36(33.4)$ \\
\hline
\end{tabular}

$S D$ standard deviation

Table 2 Statistical analysis of different models evaluated infusion is apparent (Fig. 1). For the intra-individual variability, the combined residual error model showed the lowest AIC and-2LL values, and was used as the base model. The estimated parameters of the final model are shown in Table 3.

\subsection{Evaluation of Covariates}

The results of the univariate analysis of the correlation of variables with PK parameters are shown in Table 4. There was a statistically significant association of serum creatinine with CL $(p=0.01)$ and of body weight with $V(p=0.05)$. The serum creatinine level is inversely associated with the $\mathrm{CL}$ of $\mathrm{MgSO}_{4}$ and, consequently, directly associated with the serum concentrations of magnesium. Body weight is directly associated with the $V$ and, therefore, associated with lower serum magnesium concentration. Serum levels of albumin and total protein, age, and the presence of comorbidities were not significantly associated with PK parameters.

The observed plasma concentrations showed good correlation with the population and individual plasma concentrations predicted by the final model (Fig. 2). Both population and individual weighted residuals (PWRES and IWRES, respectively) and normalized predicted errors (NPDE) showed normal distribution with near zero mean (Fig. 2). In the VPC, it can be seen that the 5th, 50th, and 95th percentiles of the observed data were close to the percentiles of the simulated data, with few outliers (Fig. 3).

The equations for $\mathrm{MgSO}_{4}$ concentration, $V$, and population CL were as follows:

$\mathrm{Mg}=$ basalMg $+(1.76+0.000552 \times$ basalMg $) \times \exp (\mathrm{CL} / V \times t)$,

with $\mathrm{CL}=\exp (0.3221-0.0814 \times$ creatinine $(\mathrm{mg} /$ $\mathrm{dL})+0.0151)$ and $V=\exp (2.5878+0.0752 \times$ weight $(\mathrm{kg})+0.404)$.

\begin{tabular}{lrrr}
\hline Structural model & \multicolumn{1}{c}{-2LL } & AIC & \multicolumn{1}{c}{ BIC } \\
\hline One compartment model with proportional error & 1028.90 & 1042.90 & 1061.68 \\
One compartment model with constant error & 1040.58 & 1052.58 & 1068.68 \\
One compartment model with combined error & 928.23 & 942.23 & 961.00 \\
Two compartment model with proportional error & 2814.27 & 2828.27 & 2847.05 \\
Two compartment model with constant error & 2129.89 & 2139.89 & 2153.30 \\
Two compartment model with combined error & 1130.02 & 1140.02 & 1153.43 \\
\hline
\end{tabular}

AIC Akaike information criteria, BIC Bayesian information criteria, $-2 L L-2 \times \log$-likelihood 

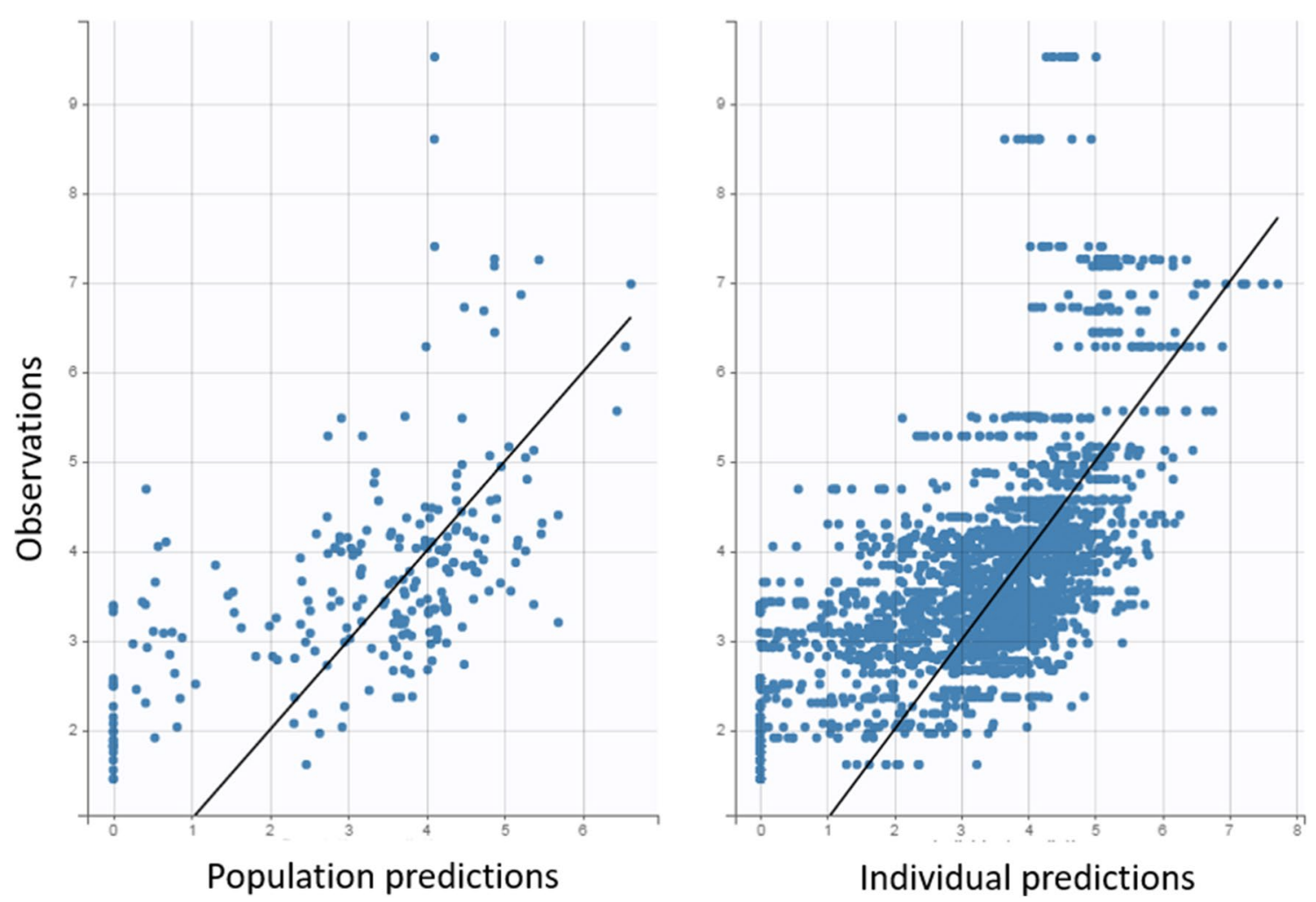

Fig. 1 Correlation between observed plasma concentration and population plasma concentration (left) and with individual predicted values (right)

Table 3 Estimated magnesium sulfate population parameters

\begin{tabular}{|c|c|c|c|c|c|}
\hline Fixed effect & Estimated & $\mathrm{RSE}^{\mathrm{a}}$ & $\%$ & $p$ value & Bootstrap \\
\hline$V \_$pop & 13.3 & 0.112 & 8.43 & & 13.3 \\
\hline CL_pop & 1.38 & 0.0239 & 17.4 & & 1.4 \\
\hline Beta_CL_creatinine_mg_dL & -0.0814 & 0.0279 & 34.2 & 0.00349 & \\
\hline Beta_ $V \_W e i t h \_k g$ & +0.0752 & 0.0187 & 28 & 0.00878 & \\
\hline Random effect & Estimated & Standard deviation & $\mathrm{CV}$ & $p$ value & Bootstrap \\
\hline Omega_ $V$ & 0.404 & 0.0777 & 19.3 & & 0.403 \\
\hline Omega_CL & 0.015 & 0.00882 & 38.5 & & 0.015 \\
\hline Residual error & Estimated & Standard deviation & $\mathrm{CV}$ & & \\
\hline$a$ & 1.76 & 0.131 & 7.41 & & \\
\hline$b$ & 0.000552 & 0.0000363 & 6.57 & & \\
\hline-2 log-likelihood & 861.73 & & & & \\
\hline AIC & 877.73 & & & & \\
\hline $\mathrm{BIC}$ & 899.12 & & & & \\
\hline
\end{tabular}

AIC Akaike information criteria, BIC Bayesian information criteria, $C L$ clearance, $C V$ coefficient of variation, $R S E$ relative standard error, $V$ distribution volume

${ }^{\mathrm{a}} \mathrm{RSE}=($ standard error/estimated value $) \times 100$ 
Table 4 Covariate analysis with the best POPPK base model

\begin{tabular}{llll}
\hline Covariate & -2LL & AIC & $p$ value \\
\hline Albumin on CL & 915.873 & 935.948 & 0.33 \\
Albumin on $V$ & 922.736 & 938.562 & 0.35 \\
Total protein on CL & 918.645 & 936.934 & 0.27 \\
Total protein on $V$ & 917.374 & 937.487 & 0.22 \\
Creatinine on CL & 861.734 & 877.734 & 0.035 \\
Creatinine on $V$ & 893.437 & 926.475 & 0.25 \\
Age on CL & 879.374 & 901.573 & 0.071 \\
Age on $V$ & 894.571 & 913.846 & 0.13 \\
Weight on CL & 886.478 & 903.478 & 0.24 \\
Weight on $V$ & 861.734 & 877.734 & 0.009 \\
Comorbidities on CL & 920.479 & 940.734 & 0.42 \\
Comorbidities on $V$ & 912.873 & 937.493 & 0.39 \\
Concomitant use of other drugs on CL & 893.468 & 932.477 & 0.40 \\
Concomitant use of other drugs on $V$ & 927.547 & 940.750 & 0.62 \\
\hline
\end{tabular}

AIC Akaike information criteria, $C L$ clearance, $P O P P K$ population pharmacokinetic, $V$ distribution volume, $-2 L L-2 \times \log$-likelihood

\section{Discussion}

$\mathrm{MgSO}_{4}$ is a medicine used in the therapy of PE and eclampsia [10-14, 16] and in fetal neuroprotection [17], but its therapeutic concentration is not well established [18]. Treatment protocols that do not consider individual variation may result in serum concentrations of magnesium above or below the reference range and increased maternal and fetal risks [8]. Therefore, a better understanding of how individual variation may influence the PK parameters could allow safer management of these patients.

This study described the $\mathrm{PK}$ of $\mathrm{MgSO}_{4}$ administered to patients with $\mathrm{PE}$ and has shown that it is significantly influenced by creatinine and body weight. The patients in this study had baseline magnesium values compatible with their gestational period $[10,13,19]$. The study reported PK data with the Zuspan regimen (4-g loading dose and infusion rate of $1 \mathrm{~g} / \mathrm{h}$ ). After $2 \mathrm{~h}$ of infusion, magnesium reached steady-state concentrations, resulting in serum values in the range of $1-2 \mathrm{mmol} / \mathrm{L}(2.43-4.9 \mathrm{mg} / \mathrm{dL})$, and after $18 \mathrm{~h}$, the serum levels showed a slight decrease. Brookfield et al. [8] observed higher steady-state concentrations $(7.2 \mathrm{mg} / \mathrm{dL})$ in $\mathrm{PE}$, but the maintenance infusion rate in their study was $2 \mathrm{~g} / \mathrm{h}$. Other authors using similar therapeutic regimens to ours have observed values closer to our data: a reported range of $1-3 \mathrm{mmol} / \mathrm{L}(2.43-7.29 \mathrm{mg} / \mathrm{dL})$ [11] and a reported range of $1.64-1.70 \mathrm{mmol} / \mathrm{L}(4.00-4.13 \mathrm{mg} / \mathrm{dL})$ [15]. Regarding the observed half-life values, they are compatible with those described by Chuan et al. [11] in pregnant women, which, incidentally, resemble the half-life of $\mathrm{MgSO}_{4}$ in nonpregnant women [20].
Despite the discrepancies between the different studies, all have a common characteristic also identified in our data: the concentration of magnesium in the steady state of many patients was above or below the reference values recommended in the literature $(2.5-3.5 \mathrm{mmol} / \mathrm{L})$ [21]. Sibai and Ramanathan [22] reported that the effective therapeutic concentration might be below the therapeutic range recommended in the literature. These authors suggested that the maintenance dose of the Zuspan regimen might be of particular interest because it was associated with a magnesium steady-state concentration lower than $2 \mathrm{mmol} / \mathrm{L}(4.9 \mathrm{mg} / \mathrm{dL})$. This was also found in our study, where a mean steady-state magnesium concentration of $1.31 \mathrm{mmol} / \mathrm{L}(3.18 \mathrm{mg} / \mathrm{dL})$ was observed. Similar to these results, another study found that $\mathrm{MgSO}_{4}$ therapy at the maintenance dose of $1 \mathrm{~g} / \mathrm{h}$ was just as effective as the 2-g maintenance dose, with fewer side effects [23].

A serum concentration range of magnesium between 2 and $3.5 \mathrm{mmol}(4.9-8.5 \mathrm{mg} / \mathrm{dL})$ is associated with increased toxicity [15]. With levels of $3.75-5 \mathrm{mmol} / \mathrm{L}(9-12 \mathrm{mg} / \mathrm{dL})$, there is loss of the patellar reflex, which is the first sign of $\mathrm{MgSO}_{4}$ toxicity. Other initial clinical manifestations of toxicity include sensation of warmth, flushing, double vision, nausea, drowsiness, slurred speech, and muscular weakness. Muscular and respiratory paralysis develop with levels of 6-7 mmol/L (15-17 mg/dL) [22-24]. In our study, we observed that even though the steady-state concentration of most patients was less than $2 \mathrm{mmol} / \mathrm{L}$, there was a therapeutic response. In addition, 7\% (8/114) of the patients presented absence of patellar reflex as a sign of toxicity, and these were those that reached serum levels of magnesium $>3.75 \mathrm{mmol} / \mathrm{L}$.

The model that allowed us to adequately describe the serum concentration of magnesium was the one-compartment model. This finding is consistent with the study by Brookfield et al. [25]. The choice of a one- or two-compartment model is dependent on the source data and the sampling scheme, with a one-compartment model usually used for sparse PK sampling, which was the case in this study, and a two-compartment model for serial PK sampling [15, 26].

Okusanya et al. [15] reported that the volume of magnesium distribution ranged between 13.65 and $49.00 \mathrm{~L}$, but the CL was similar to that for other populations (4.28-5.00 $\mathrm{L} / \mathrm{h})$. The value of $V$ was compatible with that found in the study published by Salinger et al., which was $15.6 \pm 8 \mathrm{~L}$ [27]. The estimated CL value in the population of this study is slightly below values described in the literature, which may be explained by the higher levels of serum creatinine in the evaluated patients.

During pregnancy, there is significant weight gain due to the increase in the volume of fluid and, consequently, of the $V$ [27]. This situation causes hemodilution of the magnesium ion in relation to the increase in weight, causing women who 

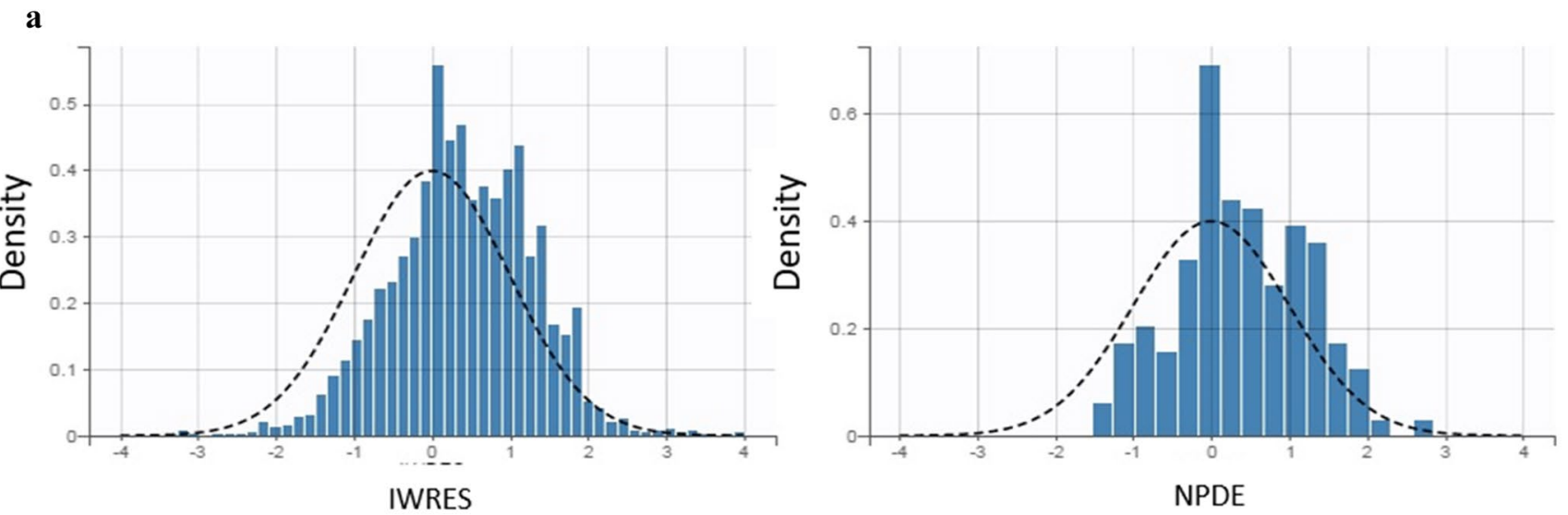

b
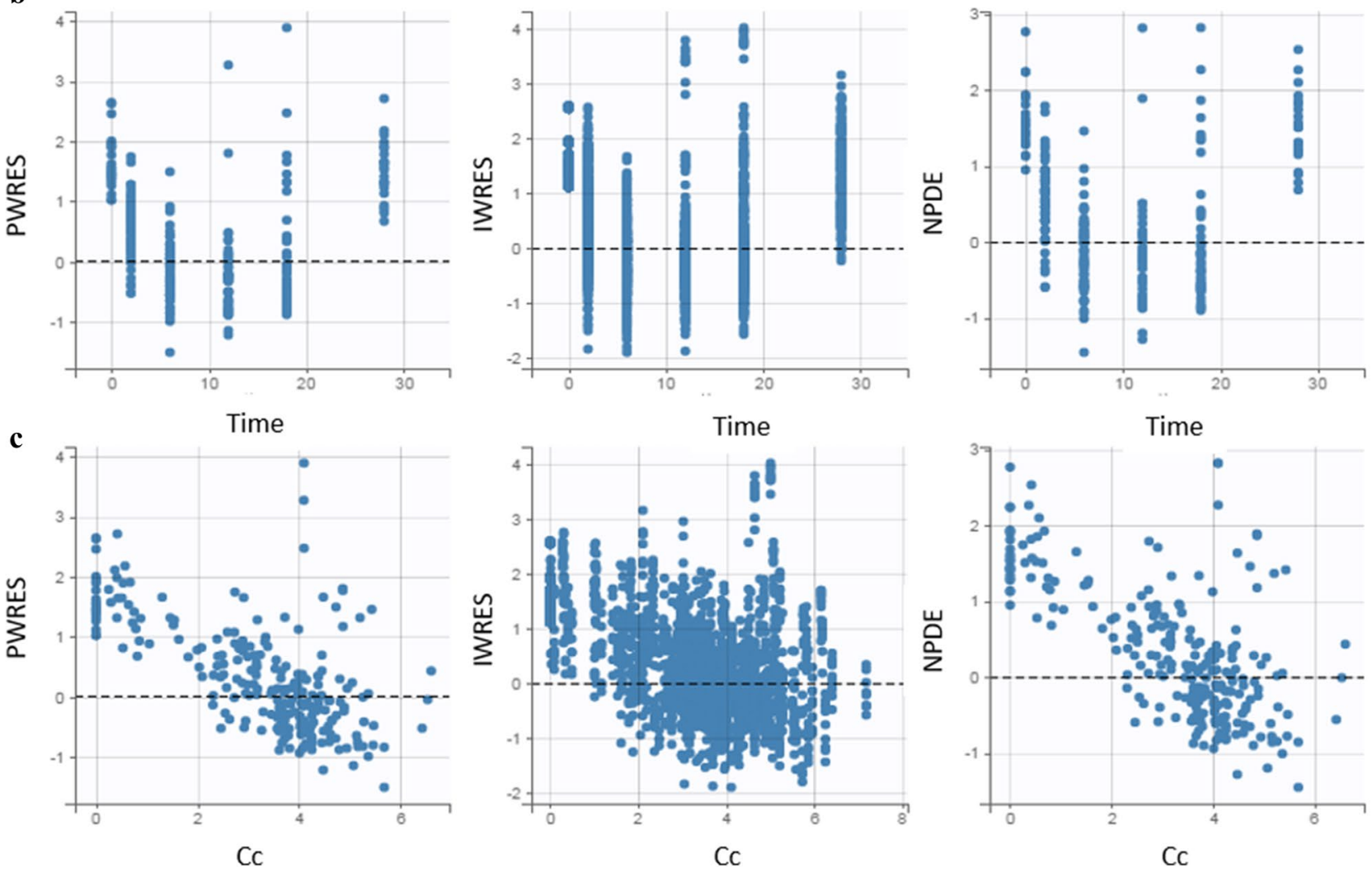

Fig. 2 Distribution of residuals. a Representation of the normal distribution of residuals. The dashed line represents the theoretical distribution defined in the developed model and the bars the distribution of the observed data. b Homogeneous distribution of weighted residuals in relation to time. $\mathbf{c}$ Homogeneous distribution of weighted residuals in relation to predicted plasma concentration. IWRES weighted individual residual, NPDE normalized predicted errors, $P W R E S$ weighted population residual weigh more to have a subtherapeutic level of serum magnesium after receiving the standard dose of $\mathrm{MgSO}_{4}$ [25]. The differences in extravascular volume due to edema associated with PE may also contribute to the variability of the $V$ [27]. Moreover, the greater the maternal weight, the greater the time to achieve steady state compared to women of lower weight [8].

Maternal adaptation to pregnancy involves dramatic changes in renal hemodynamics, including a 40-65\% increase in glomerular filtration rate during the first half of pregnancy, which is maintained during the later stages of pregnancy. $V$ is usually increased during pregnancy, especially in preeclamptic-eclamptic patients who retain more extracellular fluid. In addition, renal CL may be decreased in women with severe PE-eclampsia [8, 21].

The correlation between observational data versus the population and individual predictions (Fig. 1) showed adequate fit of the selected model, Moreover, residuals versus 
Fig. 3 Predicted versus observed concentrations of $\mathrm{MgSO}_{4}$ over infusion time (VPC). The dotted lines represent the 5th, 50th, and 95th percentiles of the simulated data. The areas indicate the range of $90 \%$ predicted associated with the 5th, 50th, and 95th percentiles of the simulated data. The blue circles correspond to the observed values and the black circles to the predicted values. $V P C$ visual predictive check

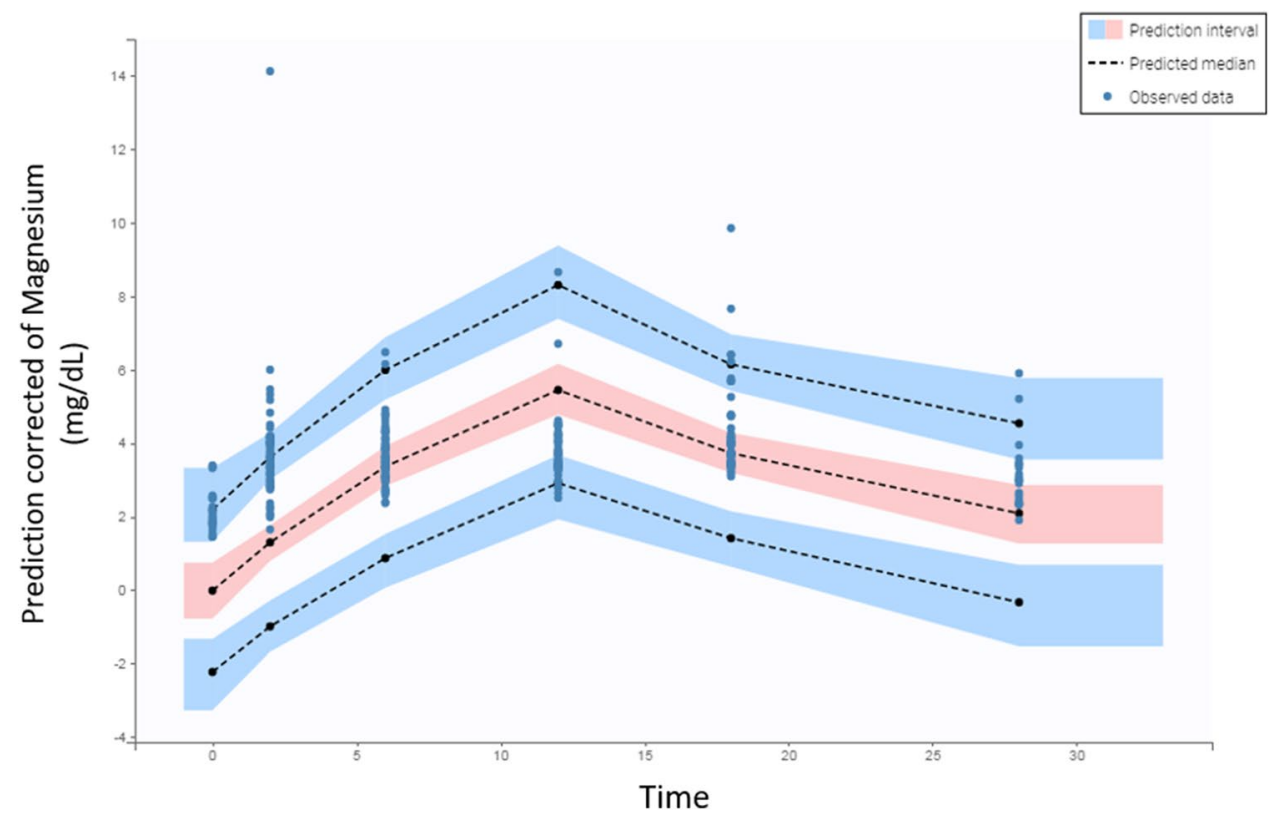

time (Fig. 2b) and residuals versus predicted concentrations (Fig. 2c) showed a fairly symmetrical distribution around zero.

Our results confirm that the variability among individuals can be clinically relevant, as shown in the PK equations obtained, which demonstrate the influence of serum weight and creatinine on the $V$ and CL, respectively. These equations were based on the univariate analysis of different continuous and categorical covariates. In clinical practice, weight and serum creatinine were shown to be important parameters that should be considered in the individualization of therapy, a recommendation also suggested by other investigators $[24,25]$ in their studies. Using the PK population model, the maintenance dose can be estimated in order to individualize the dosage for women based on their baseline characteristics, maximizing the therapeutic benefit and minimizing the toxicity of the drug, especially for women with extreme values of creatinine and weight. This maintenance dose equation is a useful tool for clinical practice.

Future research should be carried out in pregnant women and postpartum women, and should consider a greater number of different covariates (such as ionized calcium, glomerular filtration, and hematologic parameters), regimens, and treatment doses and evaluate the impact of basal magnesium on therapy. These studies should identify the factors that significantly influence the $\mathrm{PK}$ of $\mathrm{MgSO}_{4}$, allowing the development of a model that allows the individualization of therapy.

This study broadens the literature on $\mathrm{MgSO}_{4} \mathrm{PK}$ in PE, adding data on some covariates and corroborating data found in other studies. This study also had some limitations, such as there is substantially more data in the observed versus individual predicted plot than there is in the observed versus population predicted plot. These plots should have the same number of data points, so this discrepancy limits the confidence in the population PK analysis and accuracy of the model. Moreover, based on the distribution of the IWRES, the plots suggest that the individual predicted concentrations are skewed towards being greater than the observed concentrations. In addition, other limitations are the presence of endogenous magnesium before the administration of $\mathrm{MgSO}_{4}$, the need for a larger number of samples in some patients in order to optimize the development of the POPPK model, and the possibility of omission in the measurement of important covariates that were not included in the final population PK model.

\section{Conclusions}

The study described the PK of magnesium administered to pregnant women with $\mathrm{PE}$ and demonstrates that $\mathrm{PK}$ is influenced by creatinine serum concentration and the weight of the mother. The variability is due to differences in body weight, as a reflex of the $V$, and differences in serum creatinine concentration, as a reflex of the glomerular filtration rate. The profile of the Zuspan regimen indicates that the minimum effective magnesium concentration for the prophylaxis of eclampsia is lower than the generally accepted level. PK-PD studies are necessary to confirm this finding, considering that a lower therapeutic concentration would provide a lower risk of toxicity, but could not achieve the desired therapeutic effect in some patients, leading to an increase in eclampsia. Therefore, taking into account the PK alterations 
demonstrated in this study, obtaining the ideal therapeutic level of $\mathrm{MgSO}_{4}$ is related to the individualization of therapy.

Acknowledgements The authors would like to thank the clinical team for assistance in data collection.

Author contributions All authors contributed to the design of the study. The preparation of the material and the collection and analysis of the data were performed by TXdaC, FJA, MAGU, MAdaSF, RRM, and AGO. The first draft of the manuscript was written by TXdaC, and all the authors commented on the all versions of the manuscript. All authors have read and approved the final manuscript.

\section{Compliance with Ethical Standards}

Ethical approval All procedures performed in studies involving human participants were in accordance with the ethical standards of the institutional research committee comitê de ética em pesquisa do hospital universitário onofre lopes and with the 1964 Helsinki declaration and its later amendments or comparable ethical standards.

Funding This study was financed in part by Coordenação de Aperfeiçoamento de Pessoal de Nivel Superior - Brasil (CAPES) (Finance code 001).

Conflict of interest The authors, Tatiana Xavier da Costa, Francine Johansson Azeredo, Marcela Abbott Galvão Ururahy, Miguel Adelino da Silva Filho, Rand Randall Martins, and Antonio Gouveia Oliveira, declare that there is no conflict of interest.

Open Access This article is licensed under a Creative Commons Attribution-NonCommercial 4.0 International License, which permits any non-commercial use, sharing, adaptation, distribution and reproduction in any medium or format, as long as you give appropriate credit to the original author(s) and the source, provide a link to the Creative Commons licence, and indicate if changes were made. The images or other third party material in this article are included in the article's Creative Commons licence, unless indicated otherwise in a credit line to the material. If material is not included in the article's Creative Commons licence and your intended use is not permitted by statutory regulation or exceeds the permitted use, you will need to obtain permission directly from the copyright holder. To view a copy of this licence, visit http://creativecommons.org/licenses/by-nc/4.0/.

\section{References}

1. Mol BWJ, Roberts CT, Thangaratinam S, Magee LA, De GCJM, Hofmeyr GJ. Pre-eclampsia. Lancet. 2016;387:999-1011. https:// doi.org/10.1016/S0140-6736(15)00070-7.

2. El-Khayat W, Atef A, Abdelatty S, El-Semary A. A novel protocol for postpartum magnesium sulphate in severe pre-eclampsia: a randomized controlled pilot trial. J Matern Neonatal Med. 2016;29(1):154-8. https://doi.org/10.3109/14767058.2014.99191 5.

3. Kattah AG, Garovic VD. The management of hypertension in pregnancy. Adv Chronic Kidney Dis. 2013;20(3):229-39. https ://doi.org/10.1053/j.ackd.2013.01.014.

4. Rosser ML, Katz NT. Preeclampsia : an obstetrician's perspective. Adv Chronic Kidney Dis. 2013;20(3):287-96. https://doi. org/10.1053/j.ackd.2013.02.005.
5. Smith JM, Lowe RF, Fullerton J, Currie SM, Harris L, Felkerkantor E. An integrative review of the side effects related to the use of magnesium sulfate for pre-eclampsia and eclampsia management. BMC Pregnancy Childbirth. 2013;13:1-11. https://doi. org/10.1186/1471-2393-13-34.

6. WHO recommendations for Prevention and treatment of preeclampsia and eclampsia. Geneva: World Health Organization; 2011.

7. Hart LA, Sibai BM, Ic ART, Info LE. Seizures in pregnancy: epilepsy, eclampsia, and stroke. Semin Perinatol. 2013;37:207-24. https://doi.org/10.1053/j.semperi.2013.04.001.

8. Brookfield K, Su F, Drover D, Adelus M, Lyell D, Carvalho B. Pharmacokinetics of magnesium sulfate in pregnant women. Am J Obstet Gynecol. 2015;212(1):S102. https://doi.org/10.1016/j. ajog.2014.10.221.

9. Charles B. Population pharmacokinetics: an overview. Aust Prescr. 2014;37(6):210-3. https://doi.org/10.18773/austprescr .2014.078.

10. Food and Drug Administration. Guidance for industry population pharmacokinetics. Fed Regist. 1999;64(27):6663-4.

11. Chuan FS, Charles BG, Boyle RK, Rasiah L. Population pharmacokinetics of magnesium in preeclampsia. Am J Obstet Gynecol. 2001. https://doi.org/10.1067/mob.2001.116726.

12. Easterling T, Herbert M, Bracken H, Darwish E, Ramadan MC, Shaarawy S, Charles D, Abdel-Aziz T, Nasr AS, Safwal SM, Winikoff B. A randomized trial comparing the pharmacology of magnesium sulfate when used to treat severe preeclampsia with serial intravenous boluses versus a continuous intravenous infusion. BMC Pregnancy Childbirth. 2018;18(1):290.

13. Zuspan F. Treatment of severe preeclampsia and eclampsia. Clin Obstet Gynecol. 1966;9:954-72.

14. European Medicines Agency. Committee for Medicinal Products for Human Use (CHMP) guideline on reporting the results of population pharmacokinetic analyses. 2007;1-11. https://www. emea.europa.eu. Accessed 1 Nov 2019.

15. Okusanya BO, Oladapo OT, Long Q, Lumbiganon P, Carroli G, Qureshi Z, Duley L, Souza JP, Gulmezoglu AM. Clinical pharmacokinetic properties of magnesium sulfate in women with preeclampsia and eclampsia. BJOG. 2016;123:356-66. https://doi. org/10.1111/1471-0528.13753.

16. Usman S, Foo L, Tay J, Bennett PR, Lees C. Use of magnesium sulfate in preterm deliveries for neuroprotection of the neonate. Obstet Gynaecol. 2017;19:21-8. https://doi.org/10.1111/ tog. 12328 .

17. Freyer AM. Drug-prescribing challenges during pregnancy. Obstet Gynaecol Reprod Med. 2008;18(7):180-6. https://doi. org/10.1016/j.ogrm.2008.05.005.

18. Magee LA, Pels A, Helewa M, Rey E, Von Dadelszen P. Pregnancy hypertension: an international journal of women's cardiovascular health diagnosis, evaluation, and management of the hypertensive disorders of pregnancy. Int Soc Study Hypertens Pregnancy. 2014;4(2):105-45. https://doi.org/10.1016/j.pregh y.2014.01.003.

19. Long Q, Oladapo OT, Leathersich S, Vogel JP, Carroli G, Lumbiganon P, Qureshi Z, Gulmezoglu AM. Clinical practice patterns on the use of magnesium sulphate for treatment of pre-eclampsia and eclampsia: a multi-country survey. BJOG An Int J Obstet Gynaecol. 2017;124(12):1883-900. https://doi.org/10.1111/14710528.14400 .

20. Lu JF, Nightingale $\mathrm{CH}$. Magnesium sulfate in eclampsia and pre-eclampsia pharmacokinetic principles. Clin Pharmacokinet. 2000;38(4):305-14. https://doi.org/10.2165/00003088-20003 8040-00002.

21. Du L, Wenning L, Migoya E, Xu Y, Carvalho B, Brookfield K, Witjes H, Greef R, Lumbiganon P, Sangkomkamhang U, Titapant V, Duley L, Long Q, Oladapo OT. Population pharmacokinetic 
modeling to evaluate standard magnesium sulfate treatments and alternative dosing regimens for women with preeclampsia. J Clin Pharmacol. 2018. https://doi.org/10.1002/jcph.1328.

22. Sibai BM, Ramanathan J. The case for magnesium sulfate in preeclampsia-eclampsia. Int J Obstet Anesth. 1992. https://doi. org/10.1016/0959-289X(92)90024-X.

23. Pascoal ACF, Katz L, Pinto MH, Santos CA, Braga LCO, Maia $\mathrm{SB}$, Amorim MMR. Serum magnesium levels during magnesium sulfate infusion at $1 \mathrm{~g} / \mathrm{h}$ versus $2 \mathrm{~g} / \mathrm{h}$ as a maintenance dose to prevent eclampsia in women with severe preeclampsia. Medicine. 2019. https://doi.org/10.1097/MD.0000000000016779.

24. Please update reference 25 with complete details.

25. Brookfield KF, Su F, Elkomy MH, Drover DR, Lyell DJ, Carvalho B. Pharmacokinetics and placental transfer of magnesium sulfate in pregnant women. Am J Obstet Gynecol. 2016;214(6):737.e1-. e9. https://doi.org/10.1016/j.ajog.2015.12.060.

26. Smith JM, Lowe RF, Fullerton J, Currie SM, Harris L, Felkerkantor E. An integrative review of the side effects related to the use of magnesium sulfate for pre-eclampsia and eclampsia management. BMC Pregnancy Childbirth. 2013;5:13-34. https://doi. org/10.1186/1471-2393-13-34.

27. Salinger DH, Mundle S, Regi A, Bracken H, Winikoff B, Vicini P, et al. Magnesium sulphate for prevention of eclampsia: are intramuscular and intravenous regimens equivalent? A population pharmacokinetic study. BJOG. 2013. https://doi. org/10.1111/1471-0528.12222. 